\title{
Aggressive tissue aortic valve replacement in younger patients and the risk of re-replacement: Implications from microsimulation analysis
}

\author{
Neel K. Ranganath, MD, Michael S. Koeckert, MD, Deane E. Smith, MD, Kazuhiro Hisamoto, MD, \\ Didier F. Loulmet, MD, Aubrey C. Galloway, MD, and Eugene A. Grossi, MD
}

\begin{abstract}
Objective: Advances in transcatheter aortic valve replacement have led to the consideration of tissue aortic valve replacement in younger patients. Part of this enthusiasm is the presumption that younger patients would have more flexibility in future treatment options, such as a primary surgical aortic valve replacement followed later by transcatheter aortic valve replacement(s) (valve-in-valve), vice versa, or other permutations. We created a microsimulation model using published longevity of tissue valves to predict the outcomes of patients after primary tissue surgical aortic valve replacement.
\end{abstract}

Methods: The model calculated survival by incorporating annual mortality (Social Security Administration) and mortality from re-replacements (Society of Thoracic Surgeons) in patients with surgical aortic valve replacement. Freedom from reoperation for structural valve degeneration incorporated best published data to determine the annual risk of re-replacement for structural valve degeneration based on implant duration and stratified by patient age. A constant rate of re-replacement for nonstructural valve degeneration indications was also incorporated. Each simulation was performed for 50,000 individuals. Kaplan-Meier curves were generated to represent survival. All simulations were run within the MATLAB environment (The MathWorks, Inc, Natick, Mass).

Results: Earlier decades of life at primary surgical aortic valve replacement were associated with higher incidences of re-replacements and especially multiple rereplacements. For those patients receiving a primary tissue surgical aortic valve replacement at age 50 years, $57.2 \%$ will require a second valve, $18.0 \%$ will require a third valve, and $1.6 \%$ will require a fourth valve with average operative mortalities of $2.9 \%, 4.8 \%$, and $7.3 \%$, respectively. A 50 -year-old patient at primary surgical aortic valve replacement has a $13.1 \%$ chance of re-replacement before turning 60 years of age.

Conclusions: Microsimulation incorporates changing hazards to estimate the risk of aortic valve re-replacement in patients undergoing tissue surgical aortic valve replacement and may be a starting point for patient education and health care economic planning. (J Thorac Cardiovasc Surg 2019;158:39-45)

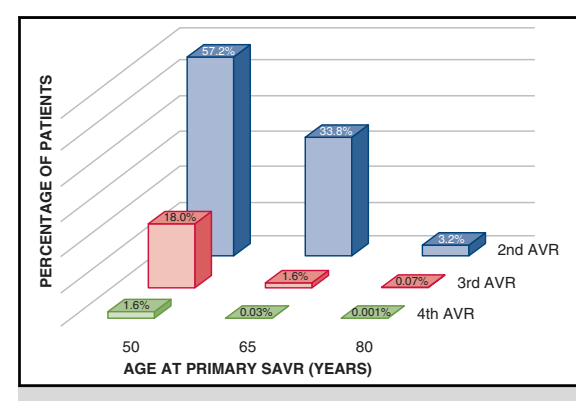

Re-replacement AVR in 50,000 simulated patients by age at primary SAVR.

Central Message

Microsimulation is a well-suited methodology for modeling the significant risk of aortic valve re-replacement in younger patients $(<65$ years old) receiving a primary tissue SAVR.

\section{Perspective}

Microsimulation models incorporate changing hazards to estimate the risk of re-replacement in patients undergoing tissue SAVR. Additional data are needed for SVD risk in TAVR and SVD risk in SAVR beyond 20 years. Microsimulation models may be a useful starting point for patient education and health care economic planning and will continue to become more accurate as additional data are incorporated.

See Commentary on page 46.

\footnotetext{
From the Division of Cardiac Surgery, Department of Cardiothoracic Surgery, NYU Langone Health, New York, NY.

Read at the 98th Annual Meeting of The American Association for Thoracic Surgery, San Diego, California, April 28-May 1, 2018.

Received for publication May 9, 2018; revisions received Sept 24, 2018; accepted for publication Oct 26, 2018; available ahead of print Feb 1, 2019.

Address for reprints: Eugene A. Grossi, MD, Department of Cardiothoracic Surgery, NYU Langone Health, 530 First Ave, Suite 9V, New York, NY 10016 (E-mail: Eugene.grossi@nyumc.org).

$0022-5223 / \$ 36.00$

Copyright (C) 2018 by The American Association for Thoracic Surgery https://doi.org/10.1016/j.jtcvs.2018.10.165
}

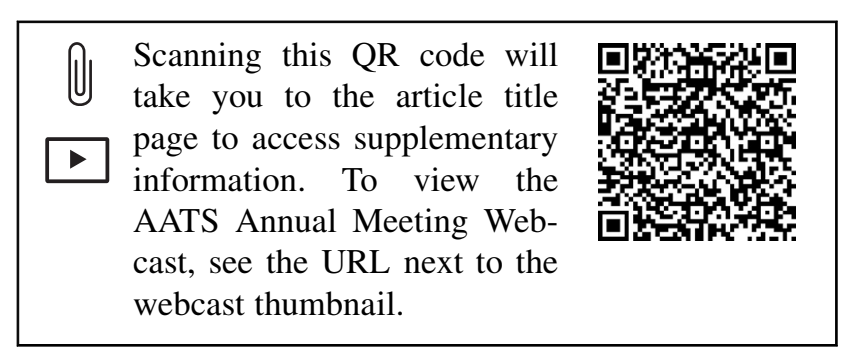




\section{Abbreviations and Acronyms}

AVR $=$ aortic valve replacement

SAVR $=$ surgical aortic valve replacement

STS = Society of Thoracic Surgeons

SVD = structural valve degeneration

TAVR $=$ transcatheter aortic valve replacement

Prosthetic valve type is a major consideration regarding surgical aortic valve replacement (SAVR). Although mechanical valves were traditionally favored in younger patients because of their better long-term durability and systemic anticoagulation is usually well tolerated, it may be lifestyle limiting for an otherwise healthy young patient. Thus, the trend in recent years has been the use of tissue valves in younger patients despite their higher risk of reoperation for structural valve degeneration (SVD) as evidenced by the current guidelines' class IIa recommendation that "for patients between 50 and 70 years of age, it is reasonable to individualize the choice of either a mechanical or bioprosthetic valve prosthesis on the basis of individual patient factors and preferences, after full discussion of the tradeoffs involved." ${ }^{1}$ Enthusiasm for tissue aortic valve replacement (AVR) in younger patients has further increased with successful advances in transcatheter aortic valve replacement (TAVR) based on the assumption that these younger patients would have greater flexibility in future treatment options.

Recent long-term data on freedom from reoperation for SVD in patients who received bioprostheses at age 50 to 60 years have been published ${ }^{2}$; such age-stratified risk data allow exploration of the trend favoring aggressive use of tissue prostheses in younger patient cohorts. Microsimulation models can use these data to predict outcomes for individual simulated patients, which can be aggregated to estimate population-level outcomes. Traditionally, microsimulations were used for health care economic analysis such as the cost-effectiveness of health screening programs. Microsimulation models differ from population-level simulation models by simulating life histories for individual patients, which allows adjusting of hazards for each individual depending on prior events, such as increasing operative mortality with each subsequent aortic valve re-replacement. $^{3-6}$ We have extended a previously described microsimulation model of aortic bioprosthesis $\mathrm{SVD}^{3}$ using published reports concerning the best functional longevity of pericardial tissue valves ${ }^{2}$ to predict outcomes for patients after primary tissue SAVR and subsequent re-replacements.

\section{MATERIALS AND METHODS}

The previously described method of Takkenberg and colleagues ${ }^{3}$ was extended by incorporating subsequent aortic valve re-replacements. We simulated 50,000 patients with prespecified age and sex at primary SAVR. Figure 1 is a schematic representation of the algorithm of our simulation. First, all patients receive a primary tissue SAVR with operative mortality based on the Society of Thoracic Surgeons (STS) risk model for primary SAVR with inputs of age, sex, and the assumption of no major comorbidities. ${ }^{7}$ Next, an annual increment in both the patient's and prosthetic's age is performed. The patient's annual risk of all-cause death adjusted by age and sex is extracted from Social Security Administration data ${ }^{8}$ and applied by a random number generator, and then the annual risk of re-replacement is calculated and likewise applied by a random number generator. The rate of re-replacement for SVD was based on long-term actuarial data for freedom from reoperation for SVD stratified by patient age at implant for the Carpentier-Edwards Perimount Valve (Edwards Lifesciences, Irvine, Calif), ${ }^{2}$ this being the most "optimistic" longevity dataset for tissue AVR. This 2659 patient cohort was $68.4 \%$ male, $13.9 \%$ age less than 60 years, included all etiologies ( $87 \%$ degenerative), excluded double

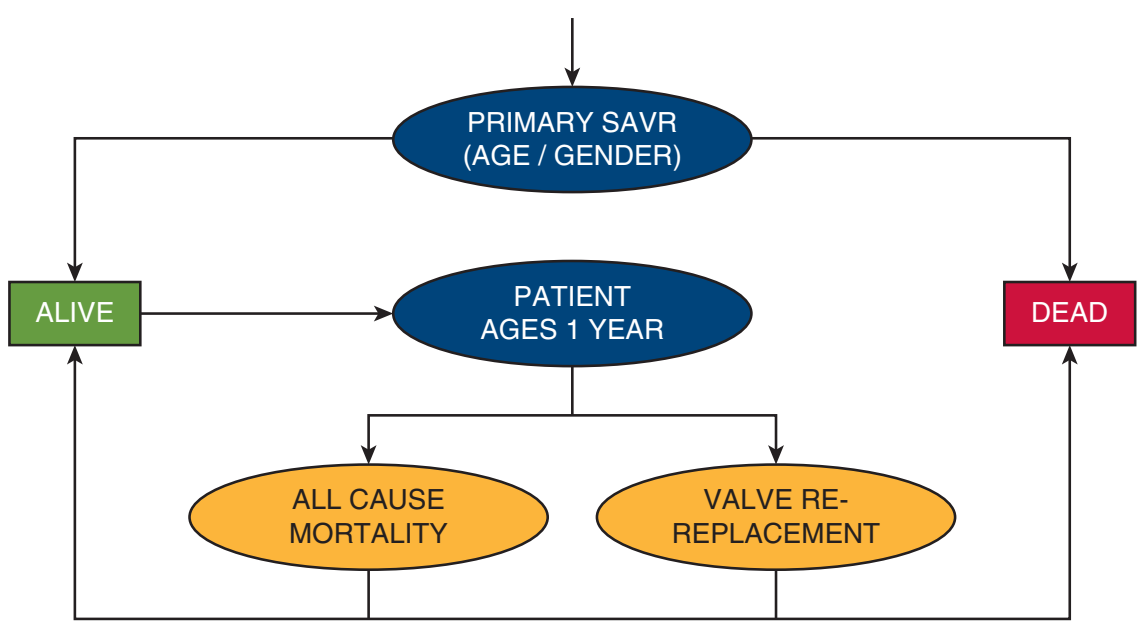

FIGURE 1. Each patient begins the simulation with a primary SAVR. Every year after incrementally increasing the age of the patient and prosthesis, we apply the annual risk of all-cause mortality (from Social Security Administration) and the annual risk of SVD requiring re-replacement. Mortality for re-replacements is derived from the STS database. SAVR, Surgical aortic valve replacement. 
valve procedures, and had a cumulative follow-up of 18,404 valve-years. These data were fitted to a reverse sigmoid function to determine the annual risk of SVD based on the duration of prosthetic implant. The annual risk of SVD was calculated as the difference between the cumulative risks of SVD for the following year and for the current year. In addition, a constant annual rate of valve explantation for non-SVD indications ${ }^{2}$ was incorporated to calculate the all-cause risk of valve explantation. If the simulated patient requires valve explantation, he/she undergoes re-replacement with the appropriate STS mortality risk incorporated. The model then reiterates for the following year. We used a cycle length of 1 year because the STS and Social Security Administration data incorporated in our model are granular only to 1-year increments in patient age. If the patient dies at any point, the simulation terminates for that patient and begins with primary SAVR for the next patient. The simulation ends when all patients have died. We used a time horizon of 119 years for the simulation by which time all patients have always died.

Our data are based on simulations of 50,000 patients at ages 50,65, and 80 years. We found that the simulation results consistently converged by using a "sample size" of 50,000 patients, and simulations of greater than 50,000 patients did not yield additional insight (Figure E1). Patient sex was selected as all male, all female, or equal distribution of male and female. Simulations were performed representing "best case" scenario (no medical comorbidities and nominate risk) and by increasing the risk of explantation for SVD by $25 \%$ and $50 \%$. Kaplan-Meier curves were generated to represent survival after primary AVR. All results are included with 95\% confidence intervals. Simulations were run within MATLAB (The MathWorks, Inc, Natick, Mass), a fourth-generation programming language and numeric computing environment.

\section{RESULTS}

For those patients receiving a primary tissue SAVR at age 50 years, $57.2 \%$ will require a second valve, $18.0 \%$ will require a third valve, and $1.6 \%$ will require a fourth valve with cumulative mortalities from re-replacements of $2.9 \%, 4.8 \%$, and $7.3 \%$, respectively, when incorporating the most favorable data. Applying a $+50 \%$ risk increase would predict $69.8 \%$ second, $29.4 \%$ third, and $3.6 \%$ fourth re-replacements for patients receiving a primary tissue SAVR at age 50 years. Table 1 presents the simulation results with risk adjustment stratified by patient age for nominate risk plus adjusters of $+25 \%$ and $+50 \%$. As expected, earlier decades of life at primary SAVR were associated with higher incidences of re-replacements and especially multiple re-replacements. Table 2 presents the impact of sex in the risk of re-replacement, with female patients consistently having higher lifetime risk of re-replacements than male patients across all age groups. Figure 2, A and $B$ present Kaplan-Meier survival plots overall and by sex for various ages at primary SAVR, demonstrating the cumulative years at risk for tissue SVD. Overall median life expectancy for a 50-year-old receiving primary SAVR was 0.3 years less than age- and sex-matched controls (Figure E2). Figure 3 illustrates the probability that a patient would require a re-replacement within 10 years of primary SAVR, which steadily decreases from $13.1 \%$ and $18.3 \%$ for the 50-year-old group to $2.5 \%$ and $3.0 \%$ for the 80 -year-old group at nominate risk and $+50 \%$ risk.

\section{DISCUSSION}

Approximately $57 \%$ of 50 -year-olds receiving a primary tissue SAVR required a second valve in their lifetime, and approximately $18 \%$ required at least 1 additional valve after that re-replacement. A sobering fact is that $13.1 \%$ of those patients would require their first rereplacement within only 10 years of their primary SAVR (Video 1). Although one could argue that a transcatheter valve-in-valve procedure ${ }^{9}$ would be an appropriate option for the first re-intervention, the unknown longevity of TAVR could dramatically impact the number of subsequent interventions required. Likewise, the geometric limitations of TAVR-in-TAVR-in-SAVR will become a limiting factor.

An interesting trend can be noted in patients who ultimately require a fourth valve. Although zero patients who receive a primary SAVR at age 80 years require a fourth valve, there is clearly an increased risk for the younger cohorts with $0.03 \%$ and $1.7 \%$ of patients who receive primary SAVR at age 65 and 50 years, respectively, requiring a fourth valve. Following this dramatic trend, we can infer by extrapolation that greater than $7 \%$ of patients who receive primary SAVR at age 40 years will require a fourth valve. Despite the significant risk of re-replacements, overall median life expectancy for a 50-year-old receiving primary SAVR was only 0.3 years less than age- and sexmatched controls, indicating that operative mortality for re-replacements is not a major cause of death after primary tissue SAVR.

Although the pericardial tissue valve data used in our simulation have demonstrated a low rate of valverelated adverse events including SVD with an expected durability beyond 20 years, our data emphasize the need for an even more durable valve in younger patients with longer life expectancy. In addition to a higher rate of SVD, the rate of valve calcification as the cause of SVD is higher in younger patients as well. Long-term outcomes of the multicenter, prospective COMMENCE trial $^{10}$ (testing novel preservation techniques in tissue valve prostheses to limit valve calcification) may reveal increased durability compared with current tissue valve prostheses.

Also, our model was based on freedom from SVD requiring reoperation before the "coming of age" of TAVR. Will we maintain the same clinical threshold for reintervention in younger patient cohorts, or will echocardiographic evidence of valve dysfunction trigger earlier reintervention, possibly even in asymptomatic patients? Current guidelines recommend surgical AVR for low- or intermediate-risk asymptomatic patients with severe aortic stenosis. ${ }^{1}$ Following through on this recommendation may dramatically increase the number of AVR to which a younger patient may be exposed. 
TABLE 1. Re-replacement aortic valve replacement for $\mathbf{5 0 , 0 0 0}$ simulated patients with equal sex distribution incorporating risk adjustments and $\mathbf{9 5} \%$ confidence intervals

\begin{tabular}{lllcl}
\hline & & Required second AVR $(\%)$ & & \multicolumn{2}{c}{ Required third AVR $(\%)$} \\
\cline { 2 - 5 } Age at primary AVR $(\mathbf{y})$ & \multicolumn{1}{c}{ No risk adjustment } & \multicolumn{1}{c}{$\mathbf{\pm 2 5 \%}$} & $\mathbf{\pm 5 0} \%$ & No risk adjustment \\
\hline 50 & $28,607 \pm 179(57.2 \pm 0.4)$ & $32,214 \pm 163(64 \pm 0.3)$ & $34,918 \pm 203(69.8 \pm 0.4)$ & $9006 \pm 157(18.0 \pm 0.3)$ \\
65 & $16,880 \pm 107(33.8 \pm 0.2)$ & $19,323 \pm 164(38.6 \pm 0.3)$ & $21,512 \pm 228(43.0 \pm 0.5)$ & $787 \pm 63(1.6 \pm 0.1)$ \\
80 & $1616 \pm 50(3.2 \pm 0.1)$ & $1776 \pm 79(3.6 \pm 0.2)$ & $1952 \pm 95(3.9 \pm 0.2)$ & $36 \pm 15(0.07 \pm 0.03)$ \\
\hline
\end{tabular}

$A V R$, Aortic valve replacement.

Consider how one would counsel an otherwise healthy 40- to 50-year-old patient who requires an AVR on his/her treatment options. If the patient is interested in receiving a tissue valve, education and planning must emphasize the high likelihood of SVD requiring reinterventions, and some of these reinterventions may not be amenable to a transcatheter procedure. Before the advent of TAVR, this patient would have required a SAVR with subsequent SAVR for each re-replacement. By introducing TAVR as an option, many unique permutations of theoretical treatment plans for primary and subsequent AVR become available. For example, the patient could receive a primary SAVR, followed by a TAVR-in-SAVR, ${ }^{9}$ before receiving a final SAVR later in life. Alternatively, the patient could receive a primary TAVR, followed by a SAVR while they are young and healthy enough to undergo the more invasive procedure, subsequently followed by a TAVR-in-SAVR. Individualized counseling would be necessary based on patient age and predicted life expectancy. Without "TAVRspecific" freedom from reintervention data, we are currently unable to model these different treatment strategies.

Finally, a newer mechanical aortic valve has demonstrated a significantly reduced risk of bleeding without a significant increase in thromboembolic complications with international normalized ratio target range of 1.5 to $2.0^{11}$; however, there are tradeoffs, including risks of bleeding and stroke in the aging patient and exclusion of those individuals from the potential benefit of future technologies (valve-in valve, advances in TAVR). This leads us to a difficult question: Should we be exposing younger patients to the risk of multiple aortic valve reinterventions to avoid the risks associated with a therapeutic international normalized ratio of 1.8 ?

\section{Study Limitations}

This simulation represents a "best case" scenario by using the most optimistic tissue valve longevity data from a single center and assuming patients do not have any major comorbidities. This is a limitation of our model because it may not be able to accurately simulate outcomes for all patient populations.

Another key limitation of our model is that we do not have reliable published data for rates of SVD requiring explantation beyond 20 years. The published data we incorporated into our model ${ }^{2}$ were fitted to a reverse sigmoid function to determine the annual risk of SVD. Figure 4 shows an example of the mathematical function created for a patient who receives a primary SAVR at age 50 years, which fits the published data very well (correlation coefficient, $r>0.99$ ) for the first 20 years after SAVR and offers reliable interpolation, but we do not know how well our extrapolation represents reality beyond that time frame. The reverse sigmoid function for SVD in Figure 4 begins with a short "latency period" for approximately 5 years after SAVR with a low annual risk. The latency period is followed by the "risk period" with a sharp increase in annual risk from approximately years 5 to 35 . On the basis of

TABLE 2. Re-replacement aortic valve replacement for $\mathbf{5 0 , 0 0 0}$ simulated patients stratified by age and sex with $95 \%$ confidence intervals

\begin{tabular}{|c|c|c|c|c|c|c|}
\hline \multirow{2}{*}{$\begin{array}{c}\text { Age at } \\
\text { primary } \\
\operatorname{AVR}(\mathbf{y})\end{array}$} & \multicolumn{2}{|c|}{ Required second AVR (\%) } & \multicolumn{2}{|c|}{ Required third AVR (\%) } & \multicolumn{2}{|c|}{ Required fourth AVR (\%) } \\
\hline & Male & Female & Male & Female & Male & Female \\
\hline 50 & $\begin{array}{l}27,703 \pm 210 \\
\quad(55.4 \pm 0.4)\end{array}$ & $\begin{array}{l}29,503 \pm 120 \\
\quad(59.0 \pm 0.2)\end{array}$ & $\begin{array}{l}8091 \pm 136 \\
\quad(16.2 \pm 0.3)\end{array}$ & $\begin{array}{l}9942 \pm 252 \\
\quad(20.0 \pm 0.5)\end{array}$ & $\begin{array}{l}677 \pm 58 \\
\quad(1.4 \pm 0.1)\end{array}$ & $\begin{array}{l}926 \pm 70 \\
\quad(1.9 \pm 0.1)\end{array}$ \\
\hline 65 & $\begin{array}{r}15,097 \pm 236 \\
\quad(30.2 \pm 0.5)\end{array}$ & $\begin{array}{r}18,575 \pm 188 \\
\quad(37.1 \pm 0.4)\end{array}$ & $\begin{array}{l}653 \pm 55 \\
\quad(1.3 \pm 0.1)\end{array}$ & $\begin{array}{l}911 \pm 40 \\
\quad(1.8 \pm 0.1)\end{array}$ & $\begin{array}{l}17 \pm 6 \\
\quad(0.03 \pm 0.01)\end{array}$ & $\begin{array}{l}23 \pm 11 \\
\quad(0.05 \pm 0.02)\end{array}$ \\
\hline 80 & $\begin{array}{l}1458 \pm 83 \\
\quad(2.9 \pm 0.2)\end{array}$ & $\begin{array}{l}1789 \pm 77 \\
\quad(3.6 \pm 0.2)\end{array}$ & $\begin{array}{l}28 \pm 11 \\
\quad(0.06 \pm 0.02)\end{array}$ & $\begin{array}{l}43 \pm 10 \\
\quad(0.09 \pm 0.02)\end{array}$ & $\begin{array}{l}0.6 \pm 1.6 \\
\quad(0.001 \pm 0.003)\end{array}$ & $\begin{array}{l}0.9 \pm 1.4 \\
\quad(0.002 \pm 0.003)\end{array}$ \\
\hline
\end{tabular}

$A V R$, Aortic valve replacement. 
TABLE 1. Continued

\begin{tabular}{|c|c|c|c|c|}
\hline \multicolumn{2}{|c|}{ Required third AVR (\%) } & \multicolumn{3}{|c|}{ Required fourth AVR (\%) } \\
\hline $\pm 25 \%$ & $\pm \mathbf{5 0} \%$ & No risk adjustment & $\pm 25 \%$ & $\pm \mathbf{5 0} \%$ \\
\hline $11,958 \pm 135(23.9 \pm 0.3)$ & $14,706 \pm 236(29.4 \pm 0.5)$ & $813 \pm 60(1.6 \pm 0.1)$ & $1258 \pm 61(2.5 \pm 0.1)$ & $1805 \pm 65(3.6 \pm 0.1)$ \\
\hline $1005 \pm 74(2.0 \pm 0.1)$ & $1226 \pm 56(2.5 \pm 0.1)$ & $19 \pm 5(0.03 \pm 0.01)$ & $33 \pm 12(0.07 \pm 0.02)$ & $40 \pm 11(0.08 \pm 0.02)$ \\
\hline $43 \pm 13(0.09 \pm 0.03)$ & $46 \pm 15(0.09 \pm 0.03)$ & $0.5 \pm 1(0.001 \pm 0.002)$ & $1.1 \pm 1.7(0.002 \pm 0.003)$ & $0.6 \pm 1.0(0.001 \pm 0.002)$ \\
\hline
\end{tabular}

currently available data, we cannot be sure how steeply the curve decreases beyond 20 years.

Takkenberg and colleagues ${ }^{3}$ had suggested that sensitivity analysis may be useful to investigate the uncertainty in rates of SVD due to heterogeneity of pooled data and variation due to patient age. However, our source data for rates of $\mathrm{SVD}^{2}$ were homogeneously collected from a single institution and stratified by patient age at implant. Therefore, sensitivity analysis would provide little additional insight for our current model. As our model expands and data from multiple sources are incorporated, sensitivity analysis will become increasingly relevant.

\section{CONCLUSIONS}

Microsimulation models incorporate changing hazards to estimate the risk of re-replacement and mortality from re-replacement in patients undergoing tissue

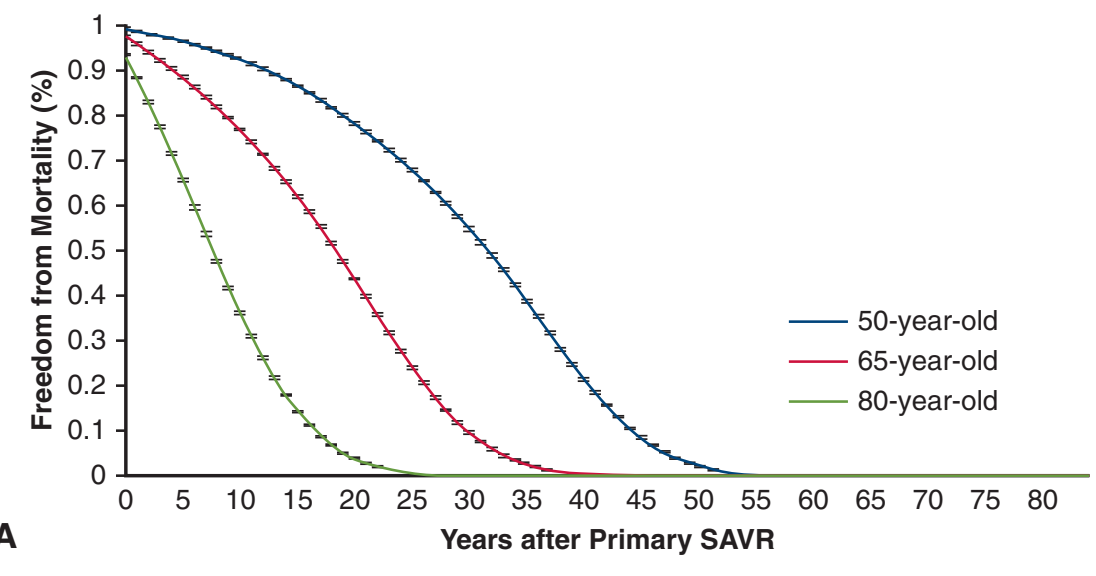

\section{A}

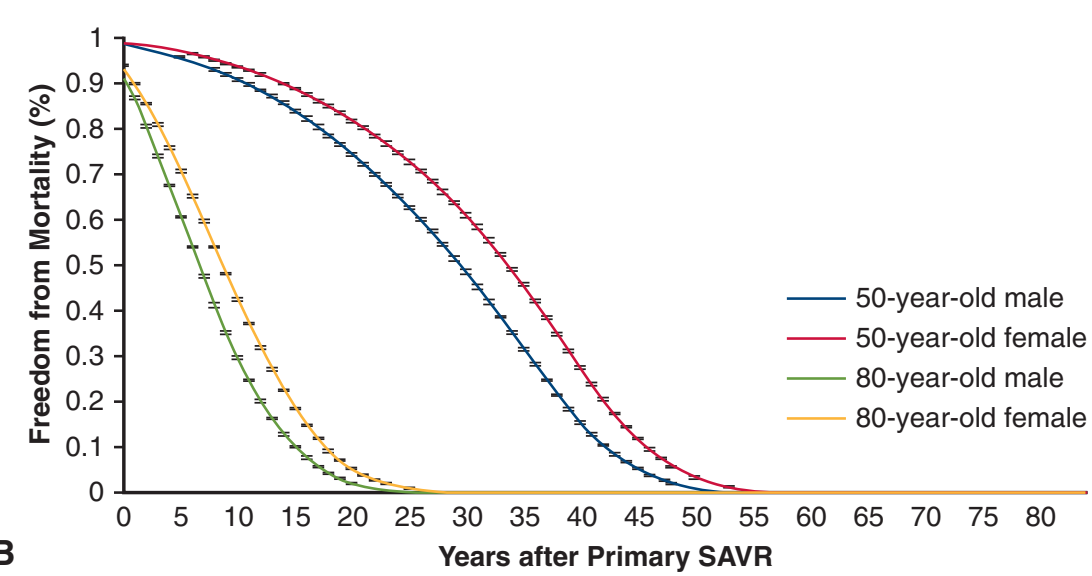

FIGURE 2. A, Patients aged 50, 65, and 80 years at primary SAVR have median life expectancies of 81.5 , 83.5 , and 87.5 years, respectively. B, Male patients who receive primary SAVR at ages 50 and 80 years have median life expectancies of 79.5 and 86.5 years, respectively. Female patients who receive primary SAVR at age 50 and 80 years have median life expectancies of 83.5 and 88.5 years, respectively. SAVR, Surgical aortic valve replacement. 


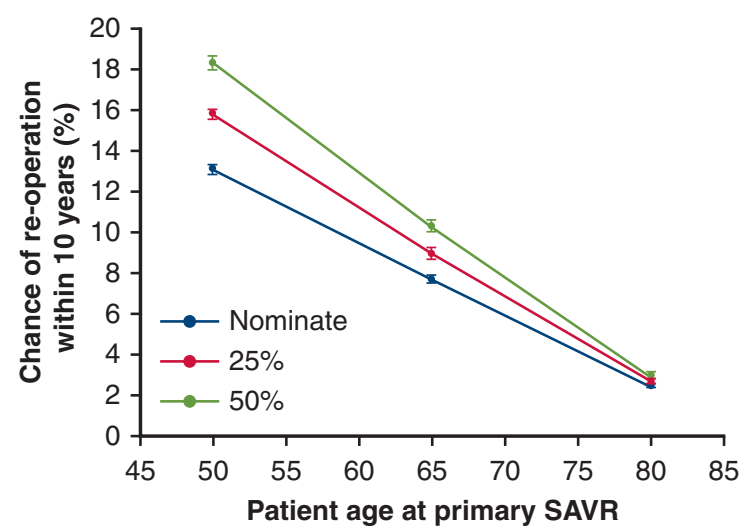

FIGURE 3. The percentage of patients requiring re-replacement within 10 years of primary SAVR monotonically decreases from $13.1 \%$ for patients aged 50 years at primary SAVR to $2.5 \%$ for patients aged 80 years at primary SAVR. SAVR, Surgical aortic valve replacement.

SAVR. Younger patients have a significant risk of requiring an aortic valve re-replacement or even multiple re-replacements. Additional data are needed for SVD risk in TAVR, SVD risk in SAVR beyond 20 years, and reinterventions on AVR prostheses indicated by echocardiographic findings in asymptomatic patients. Microsimulation models may be a useful starting point for patient education and health care economic planning and will continue to become more accurate as additional data are incorporated.

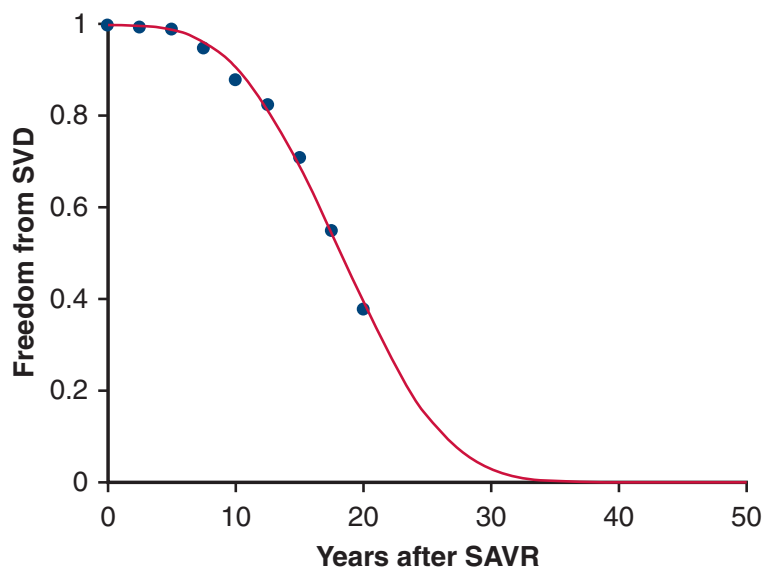

FIGURE 4. This function represents freedom from SVD in a 50-year-old patient undergoing SAVR and fits the source data very well (correlation coefficient, $r>0.99$ ). The reverse sigmoid function has an initial latency period for the first 5 years during which time SVD is rare and likely due to technical error or nonideal hemodynamics. The latency period is followed by a risk period where the majority of valves fail due to normal mechanical wear-and-tear. SAVR, Surgical aortic valve replacement; SVD, structural valve degeneration.

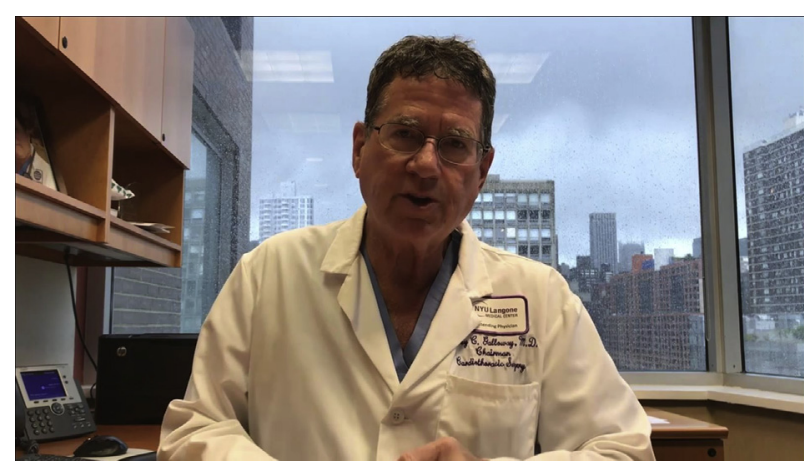

VIDEO 1. Dr Galloway discusses the use of tissue valves in younger patients ( $<65$ years) and implications of this trend obtained from microsimulation modeling. Video available at: https://www.jtcvs.org/article/ S0022-5223(18)33228-8/fulltext.

\section{Webcast}

You can watch a Webcast of this AATS meeting presentation by going to: https://aats.blob.core.windows.net/ media/18Apr30/Theater\%201\%20Booth\%20134/S61\%20$\% 20$ Annual\%20C.\%20Walton/S61_4_webcast_012023723. mp4.

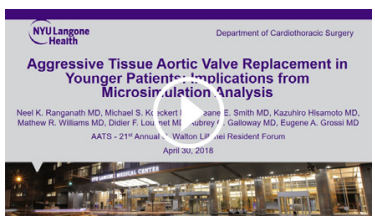

\section{Conflict of Interest Statement}

A.C.G. and E.A.G. received royalties from Medtronic and Edwards Lifesciences. All other authors have nothing to disclose with regard to commercial support. No external funding was obtained.

\section{References}

1. Nishimura RA, Otto CM, Bonow RO, Carabello BA, Erwin JP III, Fleisher LA, et al. 2017 AHA/ACC focused update of the 2014 AHA/ACC guideline for the management of patients with valvular heart disease: a report of the American College of Cardiology/American Heart Association task force on clinical practice guidelines. J Am Coll Cardiol. 2017;70:252-89.

2. Bourguignon T, Bouquiaux-Stablo AL, Candolfi P, Mirza A, Loardi C, May MA, et al. Very long-term outcomes of the Carpentier-Edwards Perimount valve in aortic position. Ann Thorac Surg. 2015;99:831-7.

3. Takkenberg JJ, Puvimanasinghe JP, Grunkemeier GL. Simulation models to predict outcome after aortic valve replacement. Ann Thorac Surg. 2003;75:1372-6.

4. van Geldorp MW, Jamieson WR, Kappetein AP, Puvimanasinghe JP, Eijkemans MJ, Grunkemeier GL, et al. Usefulness of microsimulation to translate valve performance into patient outcome: patient prognosis after aortic valve replacement with the Carpentier-Edwards supra-annular valve. J Thorac Cardiovasc Surg. 2007;134:702-9.

5. Puvimanasinghe JP, Takkenberg JJ, Eijkemans MJ, Steyerberg EW, van Herwerden LA, Grunkemeier GL, et al. Prognosis after aortic valve replacement with the Carpentier-Edwards pericardial valve: use of microsimulation. Ann Thorac Surg. 2005;80:825-31.

6. Puvimanasinghe JP, Takkenberg JJ, Eijkemans MJ, van Herwerden LA, Jamieson WR, Grunkemeier GL, et al. Comparison of Carpentier-Edwards 
pericardial and supraannular bioprostheses in aortic valve replacement. Eur $J$ Cardiothorac Surg. 2006;29:374-9.

7. Society of Thoracic Surgeons. Online STS Risk Calculator. Available at: http:// riskcalc.sts.org/stswebriskcalc/\#/calculate. Accessed February 22, 2016.

8. Social Security Administration. Actuarial Life Table. Available at: https://www. ssa.gov/oact/STATS/table4c6.html. Accessed February 22, 2016.

9. Dvir D, Webb JG, Bleiziffer S, Pasic M, Waksman R, Kodali S, et al. Transcatheter aortic valve implantation in failed bioprosthetic surgical valves. JAMA. 2014;312:162-70

10. Puskas JD, Bavaria JE, Svensson LG, Blackstone EH, Griffith B, Gammie JS, et al. The COMMENCE trial: 2-year outcomes with an aortic bioprosthesis with RESILIA tissue. Eur J Cardiothorac Surg. 2017;52:432-9.

11. Puskas J, Gerdisch M, Nichols D, Quinn R, Anderson C, Rhenman B, et al. Reduced anticoagulation after mechanical aortic valve replacement: interim results from the prospective randomized on- $\mathrm{X}$ valve anticoagulation clinical trial randomized Food and Drug Administration investigational device exemption trial. J Thorac Cardiovasc Surg. 2014;147:1202-11.

Key Words: microsimulation, aortic valve replacement, bioprosthetic valves, tissue valves, structural valve degeneration

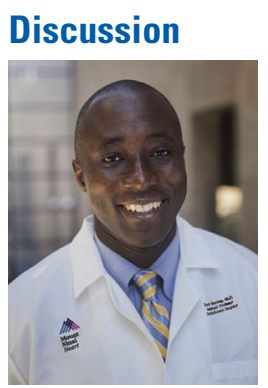

Dr P. Boateng (New York, NY). I have 3 questions. One of the premises of microsimulation is that a primary data set has to go through a validation and sensitivity analysis, validation of internal, validation if you use your own data, and then another data set to make sure that the results actually match what you include in the microsimulation. Did you do that, and if so, what did you come up with in terms of that?

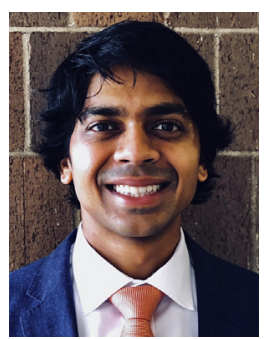

Dr Ranganath. Our model is based on a previously validated model by Grunkemeier and colleagues, but their rationale for performing sensitivity analysis was that they had heterogeneous data compiled from multiple sources. We included homogeneous long-term data from only 1 data source (Bourgignon and colleagues), and thus sensitivity analysis is not relevant to our model. We did, however, internally validate our model against our primary dataset for SVD.

Dr Boateng. My second question relates to the title of your article. You talked about bioprostheses, but the data set that you used was solely constructed from people who received the Perimount valve; that's just 1 valve type. So how do you talk to the patient who is going to get a different valve type, say a Hancock or the Mitroflow? How do extrapolate the data to the conversation with the patient going forward?

Dr Ranganath. I agree that it would be interesting to know how other valves perform within our model. We selected the Perimount valve because it had the best longterm data available, and knowing what happens $20 \pm$ years after implantation is critical for an accurate simulation. One difficulty is that the new valves are coming out faster than we can collect long-term data on older valves; we really need data of 20,25, 30 years after implantation, and new valves are coming out more quickly than that. However, we are still implanting those previous-generation valves, and many patients still have those valves in place, so we can still collect that data moving forward even though newer valves are being manufactured. Ultimately, this means that our model will not be able to simulate the newest generation of valves, because long-term data are not available for them.

Dr Boateng. My last question relates to the input of data in the microsimulation model. You mentioned in your presentation that you use an unnamed risk with no comorbidities. We know those are not real-life situations; patients have comorbidities. How do you account for not including that in your analysis?

Dr Ranganath. Unfortunately, we do not have SVD data stratified for comorbidities. Similarly, our all-cause mortality data extracted from the Social Security Administration actuarial tables are also not stratified by comorbidities. We agree that it would be interesting to know how diabetes or end-stage renal failure would affect our model, but these data are not yet available. We can, however, stratify STS mortality risk by comorbidities. Moving forward, we do plan to extend our model to incorporate the effect of comorbidities on operative mortality. Additional data will continue to make this model more accurate, and we will incorporate such data as they are published. Our goal with this project, however, was to demonstrate that microsimulation is well suited for modeling the risk of aortic valve rereplacements because of its ability to adjust hazards based on previous life events. 


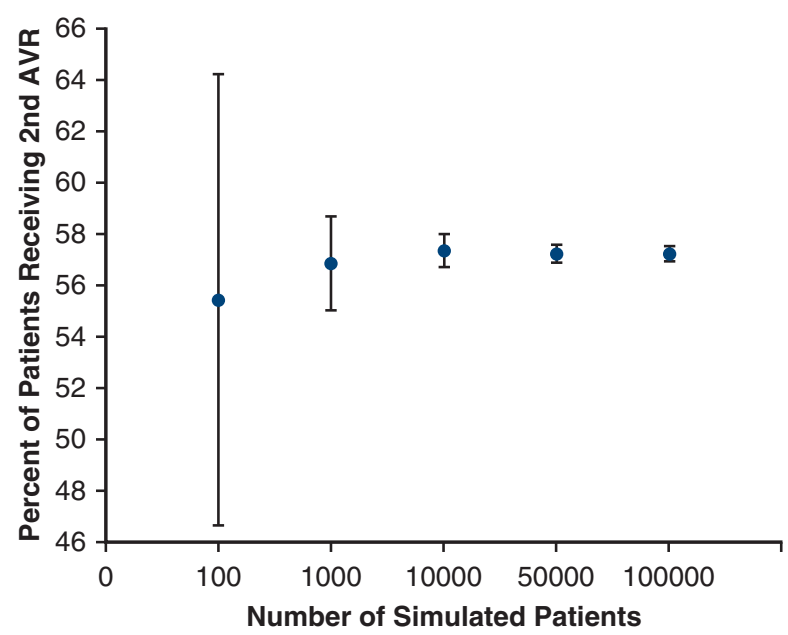

FIGURE E1. Percentages of patients receiving at least a second aortic valve replacement and $95 \%$ confidence intervals for different simulation sample sizes. These data show convergence of the simulation when using a sample size of 50,000 individuals or greater.

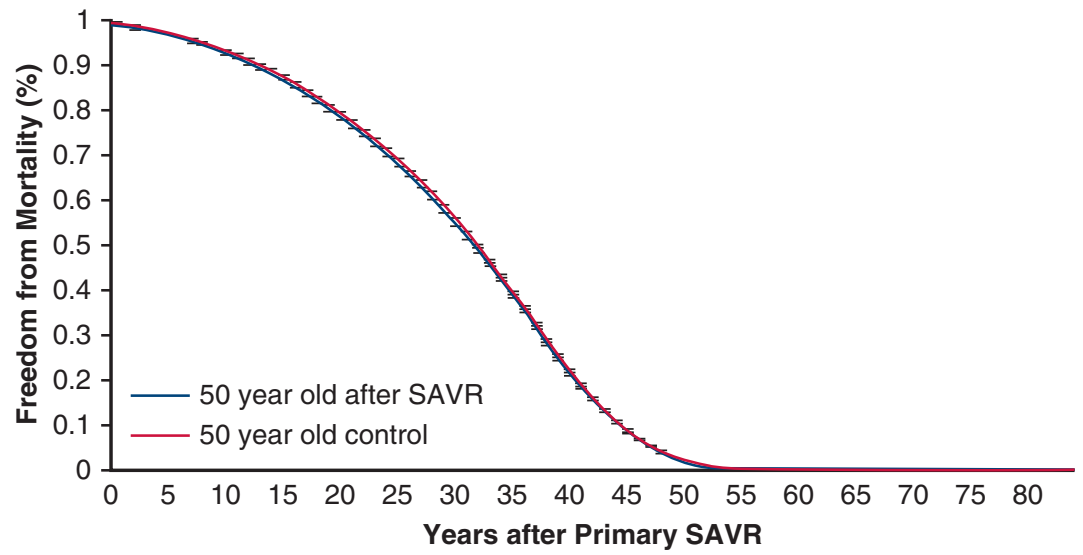

FIGURE E2. Survival curves comparing 50-year-old patients following SAVR with an age- and sex-matched control group, showing a 0.3-year difference in median survival. 